\title{
Modelo Bayesiano Hierárquico de Captura-Recaptura com Distribuição Poisson-Gama
}

M. PAULA ${ }^{1}$, Instituto de Ciências Ambientais e Desenvolvimento Sustentável, Universidade Federal da Bahia - UFBA, 45055-090 Barreiras, BA, Brasil.

C.A.R. DINIZ2, J.G. LEITE 3 , Departamento de Estatística, Universidade Federal de São Carlos - UFSCar, 13565-905 São Carlos, SP, Brasil.

Resumo. Neste artigo apresentamos um modelo bayesiano de captura-recaptura proposto por Castledine (1981) e um modelo bayesiano hierárquico com distribuição Poisson-Gama para o tamanho da população, considerando um conjunto de dados reais de captura-recaptura para uma população fechada. Apresentamos as estimativas a posteriori do parâmetro $N$, segundo os modelos bayesianos considerados bem como apresentamos algumas estimativas clássicas. Alguns aspectos sobre prioris não informativas para o modelo bayesiano hierárquico com distribuição PoissonGama são abordados e discutidos.

Palavras-chave : Poisson-Gama, captura-recaptura, população fechada.

\section{Introdução}

O processo de captura e recaptura, no caso da estimação do tamanho populacional, consiste em selecionar uma amostra de tamanho $n_{1}$ de uma população marcandoa e devolvendo-a à população. Após um certo período de tempo seleciona-se uma segunda amostra aleatória de tamanho $n_{2}$, conta-se o número de elementos marcados e marca-se os elementos não marcados, devolvendo-os à população. Após um certo período de tempo seleciona-se uma terceira amostra de tamanho $n_{3}$, conta-se o número de elementos marcados, marca-se os elementos não marcados, devolvendoos à população, e assim por diante. Esse processo é realizado $s$ vezes $(s \geq 2)$.

O interesse em estimar tamanhos de populações surgiu em meados do século XVII. Historicamente, Laplace (1786) utilizou tal processo para estimar o tamanho da população da França. Em ecologia, o primeiro pesquisador a empregar este método foi o dinamarquês Carl G. J. Petersen (1896), que estudou o fluxo migratório de peixes no mar Báltico.

As técnicas de captura-recaptura podem ser usadas para populações fechadas ou abertas. Uma população fechada é aquela em que os efeitos de nascimento, mortalidade e migração não são considerados, isto é, supõem-se que seu tamanho não

\footnotetext{
${ }^{1}$ marcelop@ufba.br

2dcad@power.ufscar.br

${ }^{3}$ leite@ufscar.br
} 
se altera durante o período de estudo (Comack, 1992). Uma população aberta é aquela que durante a realização do experimento se altera em tamanho e em composição por ocorrência de nascimentos, mortes e migrações. Com relação ao estudo de populações abertas, vários outros autores destacaram-se. Entre os mais citados estão Jolly (1965), Pollock (1991) e Schwarz e Arnason (1996). Os dados reais de captura-recaptura apresentados nesse artigo são considerados como oriundos de uma população fechada.

Neste trabalho fazemos o estudo sob o enfoque bayesiano e, devido ao fato da distribuição a posteriori conjunta ser expressa por uma expressão analítica complexa, é difícil a obtenção das distribuições a posteriori marginais dos parâmetros de inte-resse, necessárias para o cálculo de momentos a posteriori. Sendo assim, foram utilizados métodos Monte Carlo com cadeias de Markov para obtenção de amostras da distribuição a posteriori conjunta. As cadeias foram geradas através de algoritmos Gibbs Sampling e algoritmos Metropolis-Hastings, implementados utilizando-se o software R. A convergência das cadeias geradas foram diagnosticadas pelo critério do diagnóstico de Gelman-Rubin (1992) que é baseado na análise de variância, comparando-a intra e entre as cadeias geradas. Tal diagnóstico de convergência foi monitorada pelo pacote CODA - Convergence Diagnostics and Output Analysis Software for Gibbs Sampling Output.

\section{Metodologia}

Nesta seção apresentamos o modelo estatístico e determinamos a função de verossimilhança para o método de captura-recaptura com $s$ estágios de marcação $(s \geq 2)$ proposto por Castledine (1981). Denotemos por

$N$ : tamanho desconhecido da população,

$s$ : número de amostras selecionadas (épocas de captura), $s \geq 2$,

$p_{j}$ : probabilidade de qualquer animal ser capturado na $j$-ésima amostra, $j=$ $1,2, \ldots, s$ e $\mathbf{p}=\left(p_{1}, p_{2}, \ldots, p_{s}\right)$,

$n_{j}$ : número de animais capturados na $j$-ésima amostra, $j=1,2, \ldots, s$,

$m_{j}$ : número de animais marcados recapturados na $j$-ésima amostra, $j=1,2, \ldots, s$.

$M_{j}$ : é o número de elementos marcados na população anterior a $j$-ésima amostra, $j=1,2, \ldots, s$.

Vamos supor que as seguintes condições sejam verificadas:

1. a população é fechada;

2. não há animais marcados na população no início do processo, isto é, $m_{1}=0$;

3. os animais comportam-se independentemente uns dos outros;

4. as marcas não afetam a capturabilidade do animal;

5. os animais não perdem suas marcas durante o processo;

6. as épocas de amostragem são independentes. 
Neste caso, a função de verossimilhança (ver por exemplo Zacharias (2000) e Paula (2006)) é tal que

$$
L(N, \mathbf{p} \mid D)=P\left(n_{1}, m_{1} ; n_{2}, m_{2} ; \ldots ; n_{s}, m_{s} \mid \mathbf{p}, N\right) \propto\left(\begin{array}{c}
N \\
r
\end{array}\right) \prod_{j=1}^{s} p_{j}^{n_{j}}\left(1-p_{j}\right)^{N-n_{j}}
$$

onde $N \geq r, D=\left(n_{1}, m_{1} ; n_{2}, m_{2} ; \ldots ; n_{s}, m_{s}\right)$ representa os dados e

$$
r=\sum_{j=1}^{s} n_{j}-\sum_{j=1}^{s} m_{j}
$$

corresponde ao número de animais distintos capturados ao longo do processo e $0<p_{j}<1, j=1,2, \ldots, s$.

\subsection{Alguns estimadores clássicos}

Nesta seção vamos considerar dois estimadores clássicos: o estimador de máximaverossimilhança e o estimador de Schnabel, afim de fazer uma comparação com os estimadores bayesianos que serão tratados posteriormente.

\subsubsection{Estimador de máxima-verossimilhança}

Apresentamos as estimativas de máxima verossimilhança de $N$ e p, denotados por $\widehat{N}$ e $\widehat{\mathbf{p}}$ respectivamente. Tomando o logaritmo de $L(N, \mathbf{p} \mid D)$, dado em (2.1), temos

$$
\begin{aligned}
\ln L(N, \mathbf{p}) & =\ln \left[\left(\begin{array}{c}
N \\
r
\end{array}\right) \prod_{j=1}^{s} p_{j}^{n_{j}}\left(1-p_{j}\right)^{N-n_{j}}\right] \\
& =\ln \left(\begin{array}{c}
N \\
r
\end{array}\right)+\ln \left[\prod_{j=1}^{s} p_{j}^{n_{j}}\left(1-p_{j}\right)^{N-n_{j}}\right] \\
& =\ln \left(\begin{array}{c}
N \\
r
\end{array}\right)+\sum_{j=1}^{s}\left[n_{j} \ln p_{j}+\left(N-n_{j}\right) \ln \left(1-p_{j}\right)\right]
\end{aligned}
$$

onde $N \geqslant r, 0<p_{j}<1, j=1,2, \ldots, s$. Logo,

$$
\frac{\partial \ln L(N, \mathbf{p})}{\partial p_{j}}=\frac{n_{j}}{p_{j}}-\frac{N-n_{j}}{1-p_{j}}=0 \Longrightarrow \frac{n_{j}}{p_{j}}=\frac{N-n_{j}}{1-p_{j}} \Longrightarrow \widehat{p}_{j}=\frac{n_{j}}{\widehat{N}}, j=1,2, \ldots, s .
$$


Por outro lado, como $\widehat{N}$ é aproximadamente igual a solução da equação $L(N, \mathbf{p})$ $=L(N-1, \mathbf{p}), N \geqslant r+1$, temos

$$
\begin{gathered}
\left(\begin{array}{c}
N \\
r
\end{array}\right) \prod_{j=1}^{s} p_{j}^{n_{j}}\left(1-p_{j}\right)^{N-n_{j}}=\left(\begin{array}{c}
N-1 \\
r
\end{array}\right) \prod_{j=1}^{s} p_{j}^{n_{j}}\left(1-p_{j}\right)^{N-n_{j}-1}, N \geqslant r+1 \\
\Longrightarrow\left(\frac{N}{N-r}\right) \prod_{j=1}^{s}\left(1-p_{j}\right)=1, N \geqslant r+1 \\
\Longrightarrow \frac{N-r}{N}=\prod_{j=1}^{s}\left(1-p_{j}\right), N \geqslant r+1 \\
\Longrightarrow 1-\frac{r}{N}=\prod_{j=1}^{s}\left(1-p_{j}\right), N \geqslant r+1 .
\end{gathered}
$$

Assim, a estimativa de máxima verossimilhança de $\mathbf{p}=\left(p_{1}, p_{2}, \ldots, p_{s}\right)$ é $\widehat{\mathbf{p}}=$ $\left(\widehat{p}_{1}, \widehat{p}_{2}, \ldots, \widehat{p}_{s}\right)$ onde

$$
\widehat{p}_{j}=\frac{n_{j}}{\widehat{N}}, j=1,2, \ldots, s
$$

E a estimativa de máxima verossimilhança de $N, \widehat{N}$, é aproximadamente a solução da equação

$$
1-\frac{r}{N}=\prod_{j=1}^{s}\left(1-p_{j}\right), N \geqslant r+1
$$

\subsubsection{Estimador de Schnabel}

O método de Petersen, usado para o caso de 2 épocas de captura, foi estendido por Schnabel (1938) para uma série de $s$ amostras $(s \geq 2)$ cujos tamanhos são dados pelo vetor $\left(n_{1}, n_{2}, \ldots, n_{s}\right)$. Neste caso, a estimativa de $N$ é dada por

$$
\widehat{N}=\frac{\sum_{j=2}^{s} n_{j} M_{j}}{\sum_{j=2}^{s} m_{j}},
$$

onde

$n_{j}$ : é o tamanho da $j$-ésima amostra, $j=1,2, \ldots, s$.

$m_{j}$ : é o número de elementos marcados na $j$-ésima amostra, $j=1,2, \ldots, s$.

$M_{j}$ : é o número de elementos marcados na população anterior a $j$-ésima amostra, $j=1,2, \ldots, s$.

Para $s=2$, o estimador dado em (2.4) se resume no estimador de Petersen. 


\subsection{Modelo bayesiano com priori de Poisson para $\mathbf{N}$}

Nesta seção a inferência sobre $N$ será tratada sob o enfoque bayesiano, ou seja, será utilizado o conhecimento a priori tanto do tamanho populacional $N$, como do vetor de probabilidades $\mathbf{p}=\left(p_{1}, p_{2}, \ldots, p_{s}\right)$ de captura dos animais. Métodos bayesianos podem ser preferíveis uma vez que informações prévias do pesquisador são incorporadas ao modelo. Supomos que as probabilidades de captura sejam, a priori, independentes e identicamente distribuídas com $p_{j}, j=1,2, \ldots, s$, tendo distribuição Beta $(\alpha, \beta)$ com $\alpha$ e $\beta$ conhecidos $(\alpha>0, \beta>0)$, isto é

$$
\pi(\mathbf{p})=\prod_{j=1}^{s} \frac{1}{B(\alpha, \beta)} p_{j}^{\alpha-1}\left(1-p_{j}\right)^{\beta-1}
$$

e que $N$ tem distribuição a priori de Poisson truncada em zero com parâmetro $\lambda$ conhecido, $\lambda>0$, ou seja

$$
\pi(N)=\frac{e^{-\lambda} \lambda^{N}}{N !\left(1-e^{-\lambda}\right)}, N=1,2, \ldots
$$

Então, a partir da distribuição a priori dada em (2.5) e (2.6) e da função de verossimilhança dada em (2.1) temos que a distribuição a posteriori conjunta de $\mathbf{p}$ e $N$ é tal que

$$
\pi(\mathbf{p}, N \mid D) \propto \frac{\lambda^{N}}{(N-r) !} \prod_{j=1}^{s} p_{j}^{n_{j}+\alpha-1}\left(1-p_{j}\right)^{N-n_{j}+\beta-1}, 0<p_{j}<1,
$$

$j=1, \ldots, s, N \geq r$.

\subsection{Modelo hierárquico com estrutura "Poisson-Gama"}

Para a distribuição a priori do tamanho populacional $N$ com uma estrutura hierárquica é usual o tipo "Poisson-Gama" (George e Robert, 1992). Para o primeiro estágio assumimos que, dado $\lambda, N$ tem uma distribuição a priori de Poisson $\lambda$, truncada em zero dada por

$$
\pi(N)=\frac{e^{-\lambda} \lambda^{N}}{N !\left(1-e^{-\lambda}\right)}, N=1,2, \ldots
$$

Para o segundo estágio assumimos que $\lambda$ tenha distribuição Gama com hiperparâmetros $a$ e $b$ conhecidos, $a>0$ e $b>0$, dada por

$$
\pi(\lambda)=\frac{b^{a}}{\Gamma(a)} \lambda^{a-1} \exp \{-\lambda b\}, \lambda>0,
$$

Na prática, quando não temos idéia sobre o valor de $\lambda$ tomamos valores pequenos para os hiperparâmetros (por exemplo $a=b=0,0001$ ) tornando a distribuição Gama não informativa. Então da distribuição a priori dada em (2.5), da função 
de verossimilhança dada em (2.1) e da distribuição a priori dada em (2.8) e (2.9) temos que a distribuição a posteriori conjunta de $\mathbf{p}, N$ e $\lambda$ é tal que

$$
\pi(\mathbf{p}, N, \lambda \mid D) \propto \frac{\lambda^{N+a-1} e^{-\lambda(1+b)}}{\left(1-e^{-\lambda}\right)(N-r) !} \prod_{j=1}^{s} p_{j}^{n_{j}+\alpha-1}\left(1-p_{j}\right)^{N-n_{j}+\beta-1} .
$$

Da distribuição a posteriori conjunta (2.10) temos as seguintes distribuições condicionais necessárias para o algorítmo Gibbs Sampling:

A distribuição condicional de $N-r$ dados $\lambda, \mathbf{p}=\left(p_{1}, p_{2}, \ldots, p_{s}\right)$ e os dados é dada por

$$
N-r \mid \mathbf{p}, \lambda, D \sim \text { Poisson }\left(\lambda \prod_{j=1}^{s}\left(1-p_{j}\right)\right) .
$$

A distribuição condicional de $\mathbf{p}=\left(p_{1}, p_{2}, \ldots, p_{s}\right)$ dados $\lambda, N$ e os dados é dada por

$$
\pi(\mathbf{p} \mid N, \lambda, D) \propto \prod_{j=1}^{s} p_{j}^{n_{j}+\alpha-1}\left(1-p_{j}\right)^{N-n_{j}+\beta-1}, j=1,2, \ldots, s .
$$

A distribuição condicional de $\lambda$ dados $\mathbf{p}=\left(p_{1}, p_{2}, \ldots, p_{s}\right), N$ e os dados é dada por

$$
\lambda \mid N, \mathbf{p}, D \propto \frac{\lambda^{N+a-1} e^{-\lambda(1+b)}}{\left(1-e^{-\lambda}\right)} .
$$

Neste estudo foi considerado uma distribuição a priori não informativa de Jeffreys $\pi(N) \propto N^{-1}$, para o tamanho populacional $N$ (ver Smith (1991), George and Robert (1992) e Basu e Ebrahimi (2001)) e um produto de prioris não informativas $\operatorname{Beta}(0,5 ; 0,5)$ para o vetor de probabilidades $\mathbf{p}=\left(p_{1}, p_{2}, \ldots, p_{s}\right)$.

A priori não informativa de Jeffreys para o parâmetro $p, 0<p<1$, é proporcional a uma $\operatorname{Beta}(0,5 ; 0,5)$ (ver Novick and Hall (1965)). Box e Tiao (1973) discutiram as idéias de Jeffreys (1961), sobre a distribuição a priori para representar o estado de ausência de informação ou ignorância a respeito do comportamento probabilístico dos parâmetros. O estudo abrangeu os casos uniparamétricos e multiparamétricos.

A crítica mais freqüente à análise Bayesiana é que diferentes prioris conduzem a diferentes respostas. Contudo, com o interesse de encontrar "objetividade" podese usar prioris não-informativas. Se houver uma amostra pequena de dados, é necessário fazer sérias considerações para a informação a priori. Quando a amostra é grande, intervalos de confiança clássicos e intervalo de credibilidade Bayesiano serão quase idênticos numericamente. Para mais informações sobre prioris não informativas ver Novick and Hall (1965), Jeffreys (1961), Box e Tiao (1973) e Yang et. al $(1994,1995,1996)$.

\section{Resultados e Discussão}

Foi feito um estudo para estimar o número de peixes (Sunfish) no Lago Gordy, Indiana (USA), em $s=14$ épocas de capturas (Gerking, 1953) onde podemos notar 
um grande número de ocasiões de amostragem e um pequeno número de unidades de captura observadas em cada ocasião amostral.

Os dados referentes a tal experimento foram abordados por vários autores, entre eles, Ricker $(1958,1975)$ ilustrando métodos clássicos para estudos de capturarecaptura e por Castledine (1981), Smith (1988) e Wang (2002) para ilustrar métodos bayesianos. As mortes decorrentes do processo de amostragem foram desconsideradas, caracterizando assim uma população fechada. A Tabela 1 mostra os dados reais de captura-recaptura (Castledine, 1981).

Tabela 1: Dados reais de captura-recaptura.

\begin{tabular}{|c|c|c|c|c|c|c|c|c|c|c|c|c|c|c|}
\hline \multicolumn{10}{|c|}{ Peixes (Sunfish) capturados no Lago Gordy, Indiana (USA). } \\
\hline \hline$j$ & 1 & 2 & 3 & 4 & 5 & 6 & 7 & 8 & 9 & 10 & 11 & 12 & 13 & 14 \\
\hline$n_{j}$ & 10 & 27 & 17 & 7 & 1 & 5 & 6 & 15 & 9 & 18 & 16 & 5 & 7 & 19 \\
\hline$m_{j}$ & 0 & 0 & 0 & 0 & 0 & 0 & 2 & 1 & 5 & 5 & 4 & 2 & 2 & 3 \\
\hline$M_{j}$ & 0 & 10 & 37 & 54 & 61 & 62 & 67 & 71 & 85 & 89 & 102 & 114 & 117 & 122 \\
\hline
\end{tabular}

Temos então a estatística $r=\sum_{j=1}^{14} n_{j}-\sum_{j=1}^{14} m_{j}=138$. A tabela 2 mostra as estimativas clássicas de máxima-verossimilhança e de Schnabel para este conjunto de dados, segundo as expressões (2.3) e (2.4) respectivamente.

Tabela 2: Estimativas clássicas.

\begin{tabular}{|l|l|}
\hline Estimativa de máxima-verossimilhança & $\widehat{N}=449$ \\
\hline Estimativa de Schnabel & $\widehat{N}=451$ \\
\hline
\end{tabular}

\subsection{Modelo bayesiano com priori de Jeffreys para o tama- nho populacional}

Como os tamanhos das amostras obtidas foram significativamente diferentes, assumimos que as probabilidades de captura são diferentes para cada uma das $s=14$ épocas de captura. Foram geradas duas cadeias paralelas de 50000 iterações cada descartando as primeiras 10000 e considerando um salto de tamanho 10, gerando assim uma amostra final de tamanho 4000 da distribuição a posteriori conjunta de $N$ e $\mathbf{p}=\left(p_{1}, p_{2}, \ldots, p_{14}\right)$. A Tabela 3 mostra a estimativa a posteriori do parâmetro $N$. 
Tabela 3: Estimativa de $N$.

\begin{tabular}{|c|c|c|}
\hline Parâmetro & $E(N \mid D)$ & Int. Cred. (95\%) \\
\hline \hline$N$ & 378 & $(287 ; 506)$ \\
\hline
\end{tabular}

\subsection{Modelo bayesiano com priori informativa de Poisson para o tamanho populacional}

Nesta seção foi considerado uma distribuição a priori informativa de Poisson truncada em zero para o tamanho populacional $N$ e um produto de prioris não informativas $\operatorname{Beta}(0,5 ; 0,5)$ para o vetor de probabilidades $\mathbf{p}=\left(p_{1}, p_{2}, \ldots, p_{14}\right)$. Afim de verificar o comportamento das estimativas a posteriori do tamanho populacional, foi atribuído diferentes valores para o hiperparâmetro $\lambda$. Foram geradas duas cadeias paralelas de 50000 iterações cada descartando as primeiras 10000 e considerando um salto de tamanho 10, gerando assim uma amostra final de tamanho 4000 da distribuição a posteriori conjunta de $N$ e $\mathbf{p}=\left(p_{1}, p_{2}, \ldots, p_{14}\right)$. A Tabela 4 mostra as estimativas dos parâmetros.

Tabela 4: Estimativas de $N$ para diferentes valores de $\lambda$.

\begin{tabular}{|l|c|c|c|c|c|}
\hline & $E(N \mid D)$ & Int. Cred. $(95 \%)$ & & $E(N \mid D)$ & Int. Cred. (95\%) \\
\hline \hline$\lambda=0,1$ & 138 & $(138 ; 139)$ & $\lambda=250$ & 264 & $(238 ; 292)$ \\
\hline$\lambda=1$ & 138 & $(138 ; 140)$ & $\lambda=275$ & 283 & $(255 ; 314)$ \\
\hline$\lambda=25$ & 145 & $(140 ; 151)$ & $\lambda=300$ & 304 & $(274 ; 334)$ \\
\hline$\lambda=50$ & 153 & $(146 ; 162)$ & $\lambda=325$ & 325 & $(293 ; 359)$ \\
\hline$\lambda=75$ & 162 & $(152 ; 173)$ & $\lambda=350$ & 347 & $(313 ; 382)$ \\
\hline$\lambda=100$ & 173 & $(160 ; 186)$ & $\lambda=375$ & 369 & $(333 ; 407)$ \\
\hline$\lambda=125$ & 184 & $(169 ; 201)$ & $\lambda=400$ & 391 & $(354 ; 429)$ \\
\hline$\lambda=150$ & 197 & $(180 ; 216)$ & $\lambda=425$ & 414 & $(374 ; 454)$ \\
\hline$\lambda=175$ & 212 & $(193 ; 233)$ & $\lambda=450$ & 437 & $(397 ; 477)$ \\
\hline$\lambda=200$ & 229 & $(209 ; 251)$ & $\lambda=475$ & 460 & $(419 ; 504)$ \\
\hline$\lambda=225$ & 246 & $(223 ; 271)$ & $\lambda=500$ & 484 & $(442 ; 528)$ \\
\hline
\end{tabular}

Podemos observar pela tabela 4 que a informação a priori do hiperparâmetro $\lambda$ predomina a informação dos dados sobre o parâmetro $N$ quando a estatística $r$, definida em (2.2), é pequena. No entanto, a medida que vamos aumentando o valor do hiperparâmetro $\lambda$ da distribuição a priori da Poisson as estimativas a posteriori do parâmetro $N$ se aproximam de $\lambda$ (média da priori Poisson), e as amplitudes dos intervalos de credibilidade também aumentam. Este fato mostra que quando usamos uma distribuição a priori informativa de Poisson para o tamanho populacional, devemos ter uma boa idéia do parâmetro $\lambda$, caso contrário as estimativas $a$ posteriori dos parâmetros do modelo serão significativamente comprometidas. 


\subsection{Modelo bayesiano hierárquico com estrutura Poisson- gama para o tamanho populacional}

Fizemos um estudo considerando um modelo bayesiano hierárquico e atribuímos uma distribuição a priori de Poisson de parâmetro $\lambda$ para o tamanho populacional $N$. Para $\lambda$ atribuímos uma distribuição a priori $\operatorname{Gama}(a, b)$ onde atribuímos diferentes valores para os hiperparâmetros $(a, b)$ de tal forma que a variância a priori fosse consideravelmente pequena (informativa: $\operatorname{Var}(\lambda)=10^{-4}$ ) até consideravelmente grande (não-informativa: $\operatorname{Var}(\lambda)=10^{10}$ ). Fixada a esperança a priori de lambda, nossa análise se prendeu aos valores da variância a priori de lambda e seus efeitos nas estimativas a posteriori de $N$. Para o vetor de probabilidades de captura $\mathbf{p}=\left(p_{1}, p_{2}, \ldots, p_{14}\right)$ atribuímos uma distribuição a priori não informativa Beta com parâmetros $\alpha=0,5$ e $\beta=0,5$, ou seja, usamos a priori de Jeffreys.

Foram geradas duas cadeias paralelas de 50000 iterações cada descartando as primeiras 10000 e considerando um salto de tamanho 10, construindo assim uma amostra final de tamanho 4000 da distribuição a posteriori conjunta de $N, \lambda$ e $\mathbf{p}=\left(p_{1}, p_{2}, \ldots, p_{14}\right)$. A Tabela 5 mostra as estimativas a posteriori dos parâmetros $N$ e $\lambda$.

Tabela 5: Estimativas de $N$ e $\lambda$ considerando diferentes valores para $a$ e $b$.

\begin{tabular}{|c|c|c|c|c|c|c|c|}
\hline $\begin{array}{c}\text { Hiperparâmetros } \\
\text { da } \begin{array}{c}\text { priori } \\
\text { de } \lambda\end{array}\end{array}$ & $\begin{array}{c}\text { Esperança } \\
\text { a priori } \\
\text { de } \lambda\end{array}$ & $\begin{array}{c}\text { Variância } \\
\text { a priori } \\
\text { de } \lambda\end{array}$ & \multicolumn{2}{|c|}{ Parâmetro $N$} & \multicolumn{2}{|c|}{ Parâmetro $\lambda$} \\
\hline \hline \multicolumn{1}{|c|}{$a$} & $b$ & $a / b$ & $a / b^{2}$ & $E(N \mid D)$ & $\begin{array}{c}\text { Int.Cred. } \\
(95 \%)\end{array}$ & $\begin{array}{c}\text { Int.Cred. } \\
(\lambda \mid D)\end{array}$ & $(95 \%)$ \\
\hline $10^{4}$ & $10^{4}$ & 1 & $10^{-4}$ & 138 & $(138 ; 140)$ & 1,01 & $(0,99 ; 1,03)$ \\
\hline $10^{3}$ & $10^{3}$ & 1 & $10^{-3}$ & 138 & $(138 ; 140)$ & 1,1 & $(1,1 ; 1,2)$ \\
\hline $10^{2}$ & $10^{2}$ & 1 & $10^{-2}$ & 139 & $(138 ; 141)$ & 2,4 & $(2,1 ; 2,7)$ \\
\hline $10^{1}$ & $10^{1}$ & 1 & $10^{-1}$ & 142 & $(139 ; 146)$ & 14 & $(12 ; 16)$ \\
\hline 1 & 1 & 1 & 1 & 166 & $(154 ; 179)$ & 83 & $(70 ; 98)$ \\
\hline $10^{-1}$ & $10^{-1}$ & 1 & $10^{-1}$ & 244 & $(208 ; 288)$ & 223 & $(178 ; 272)$ \\
\hline $10^{-2}$ & $10^{-2}$ & 1 & $10^{2}$ & 314 & $(249 ; 406)$ & 311 & $(236 ; 412)$ \\
\hline $10^{-3}$ & $10^{-3}$ & 1 & $10^{3}$ & 378 & $(283 ; 516)$ & 378 & $(275 ; 523)$ \\
\hline $10^{-4}$ & $10^{-4}$ & 1 & $10^{4}$ & 382 & $(287 ; 516)$ & 382 & $(278 ; 529)$ \\
\hline $10^{-5}$ & $10^{-5}$ & 1 & $10^{5}$ & 383 & $(285 ; 525)$ & 383 & $(278 ; 532)$ \\
\hline $10^{-6}$ & $10^{-6}$ & 1 & $10^{6}$ & 383 & $(287 ; 522)$ & 384 & $(279 ; 534)$ \\
\hline $10^{-7}$ & $10^{-7}$ & 1 & $10^{7}$ & 383 & $(287 ; 522)$ & 384 & $(279 ; 534)$ \\
\hline $10^{-8}$ & $10^{-8}$ & 1 & $10^{8}$ & 383 & $(287 ; 522)$ & 384 & $(279 ; 534)$ \\
\hline $10^{-9}$ & $10^{-9}$ & 1 & $10^{9}$ & 383 & $(287 ; 522)$ & 384 & $(279 ; 534)$ \\
\hline $10^{-10}$ & $10^{-10}$ & 1 & $10^{10}$ & 383 & $(287 ; 522)$ & 384 & $(279 ; 534)$ \\
\hline
\end{tabular}

Podemos observar pela Tabela 5 que a medida que aumentamos a variância $a$ 
priori do parâmetro $\lambda$ tornando a distribuição não-informativa, as estimativas $a$ posteriori tanto de $N$ quanto de $\lambda$ se aproximam e se estabilizam em valores bem próximos aos valores obtidos onde utilizamos a priori de Jeffreys para $N$, dado pela tabela 3. Além disso podemos notar que a partir de uma variância acima de $10^{4}$ as estimativas de $N$ e $\lambda$ bem como seus respectivos intervalos de credibilidade possuem praticamente o mesmo valor.

\section{Conclusões}

Os estudos de simulação mostraram que quando utilizamos um modelo bayesiano hierárquico onde atribuímos uma distribuição Poisson de parâmetro $\lambda$ para o tamanho populacional $N$ e para $\lambda$ atribuímos uma priori Gama $(a, b)$ não informativa, as estimativas a posteriori não apresentam diferenças com relação ao modelo onde usamos Jeffreys $\pi(N) \propto N^{-1}$. Além disso, a priori gama não-informativa para $\lambda$ forneceu uma estimativa mais próxima das estimativas clássicas de máximaverossimilhança e de Schnabel.

Quando usamos uma distribuição a priori informativa de Poisson para o tamanho populacional, devemos ter uma boa idéia do parâmetro $\lambda$, caso contrário as estimativas a posteriori dos parâmetros do modelo serão significativamente comprometidas.

Nesse sentido, uma alternativa para estes casos é adotar tal estrutura hierárquica numa situação onde não temos informação a priori disponível a respeito do tamanho populacional.

Abstract. In this paper we present a bayesian model considered by Castledine (1981) and the hierarchical bayesian model with Poisson-Gama distribution for the population size with respect to parameter $N$ posteriori using real data of a closed population capture-recaptures. We present the posteriori estimates and classical estimates for the parameter $N$. Some aspect about non informative prioris for the hierarchical bayesian model with Poisson-Gama distribution are considered.

\section{Referências}

[1] S. Basu, N. Ebrahimi, Bayesian capture-recapture methods for error detection and estimation of population size heterogeneity and dependence, Biometrika, 88 (2001), 269-279.

[2] G.E.P. Box, G.C. Tiao, "Bayesian Inference in Statistical Analysis", AddisonWesley Series in Behavioral Science, John Wiley \& Sons, New York, 1973.

[3] B.A. Castledine, Bayesian analysis of multiple-recapture sampling for a closed population, Biometrika, 68 (1981), 197-210.

[4] D.G. Comack, Internal estimation for mark-recapture studies of closed populations, Biometrics, 48 (1992), 567-76. 
[5] A. Gelman, D. Rubin, Inference from iterative simulation using multiple sequences, Statistical Science, 7 (1992), 457-511.

[6] E.I. George, C.P. Robert, Capture-recapture estimation via Gibbs sampling. Biometrika. 79, No. 4 (1992), 677-83.

[7] S.D. Gerking, Vital Statistics of the fish population of Gordy Lake, Indiana, Transactions of the American Fisheries Society, 82 (1953), 48-67.

[8] R. Ihaka, R. Gentleman, R: A language for data analysis and graphics, Journal of Computational and Graphical Statistics, 5, No. 3 (1996), 299-314.

[9] H. Jeffreys, "Theory of Probability", Oxford University Press, 3rd edition, 1961.

[10] G.M. Jolly, Explicit estimates from capture-recapture data with both death and immigration - stochastic model, Biometrika, 52 (1965), 225-47.

[11] P.S. Laplace, Sur les naissances, les mariages et les morts, in "Histoire de L 'Académie Royale des Sciences", Paris, pp. 693, (1786).

[12] W.R. Novick, W.J. Hall, A Bayesian indifference procedure, Journal American Statistical Association, 60 (1965), 1104-1117.

[13] M. Paula, "Um enfoque Bayesiano do modelo de captura-recaptura na presença de covariáveis", Dissertação de mestrado, Universidade Federal de São Carlos, 2006.

[14] C.G.J. Petersen, The yearly immigration of young plaice into Limfjord from the German sea, etc, Rept., Danish Biol. Stn., 6 (1896), 1-48.

[15] K.H. Pollock, Modeling capture-recapture, and removal statistics for estimation of demographic parameters for fish and wildlife populations: Pas, present, and future, Journal American Statistical Association, 86 (1991), 225-38.

[16] W.E. Ricker, Handbook of Computations for Biological Statistics of Fish populations, Bull. Fish. Bd. Canada, 119 (1958), 300p.

[17] W.E. Ricker, "Computation and Interpretation of Biological Statistics of Fish Population", Ottawa: Dept. of the Environment, Fisheries and Marine Service, 382p, 1975.

[18] Z.E. Schnabel, The estimation of the total fish population of a lake, Am. Math. Monthly, 45 (1938), 348-52.

[19] C.J. Schwarz, A.N. Arnason, A general methodology for the analysis of capturerecapture experiments in open populations, Biometrics, 52 (1996), 860-73.

[20] P.J. Smith, Bayesian methods for multiple capture-recapture surveys, Biometrics, 44 (1988), 1177-1189. 
[21] P.J. Smith, Bayesian analysis for a multiple capture-recapture model, Biometrika, 78 (1991), 399-408.

[22] X. Wang, "Bayesian Analysis of Capture-recapture Models", Ph.D. Dissertation, University of Missouri, Columbia. 2002.

[23] R. Yang, J.O. Berger, Estimation of a covariance matrix using the reference prior, Annal. Statist., 22, No. 3 (1994), 1195-1211.

[24] R. Yang, M.H. Chen, Bayesian analysis for random coefficient regression models using noninformative priors, Journal Multivariate Analysis, 55, No. 2 (1995), $283-311$.

[25] R. Yang, D. Pyne, "Bayesian analysis with mixed model in unbalanced case", Ph.D. Dissertation, Purdue University, 1996.

[26] H. P. Zacharias, "Aplicação do algoritmo Gibbs sampling no processo de captura-recaptura", Dissertação de mestrado, Universidade Federal de São Carlos, 2000. 